\title{
Angiología
}

www.elsevier.es/angiologia

CARTA CIENTÍFICA

\section{Complicación de la retirada percutánea de un fragmento de catéter mediante triple lazo Goose-neck}

\section{Complication of the Percutaneous Retrieval of a Catheter Fragment Using a Triple Loop Goose Neck Snare}

\author{
A. Revilla Calavia*, E.M. San Norberto Garcia, M.A. Ibañez Maraña, V. Gutierrez Alonso \\ y C. Vaquero Puerta
}

Servicio de Angiología y Cirugía Vascular, Hospital Clínico Universitario de Valladolid, Valladolid, España

Recibido el 13 de diciembre de 2010; aceptado el 14 de mayo de 2011

Disponible en Internet el 14 de julio de 2011

La fractura de un reservorio (porth-catheter) colocado en la vena subclavia y la consecuente embolización del fragmento es una entidad rara, pero potencialmente grave ${ }^{1}$. La mayoría de los casos publicados se han desarrollado como consecuencia del síndrome de pinzamiento costoclavicular ${ }^{2}$. Existen descritas distintas técnicas para recuperar fragmentos de catéteres, como son la utilización de pinzas de biopsias, cestas de cálculos, guías tip-deflecting o la utilización de lazos $^{3}$. Estos últimos se han descrito como un método útil, altamente efectivo y sin complicaciones.

Presentamos el caso de un varón de 48 años que ingresa por presentar mal funcionamiento del catéter venoso central. Es portador de un reservorio por un cuadro de malnutrición debido a un síndrome de malabsorción. El reservorio, de $8 \mathrm{Fr}$ de diámetro, se había colocado por vía percutánea en la subclavia derecha dos meses atrás. El paciente presentaba dolor en la cara anterior del tórax durante la administración de la nutrición parenteral. El examen con fluoroscopia demostró la fractura del catéter y un fragmento libre en la cava inferior.

Con anestesia local y por abordaje inguinal derecho se colocó un introductor de $12 \mathrm{Fr}$, y con la ayuda de un lazo (Entrio ${ }^{\circledR}$, Bard, $18-20 \mathrm{~mm}$ ) se atrapó el cuerpo extraño (fig. 1). En el momento de llegar el fragmento al

\footnotetext{
* Autor para correspondencia.

Correo electrónico: alvarorevilla@yahoo.es (A.R. Calavia).
}

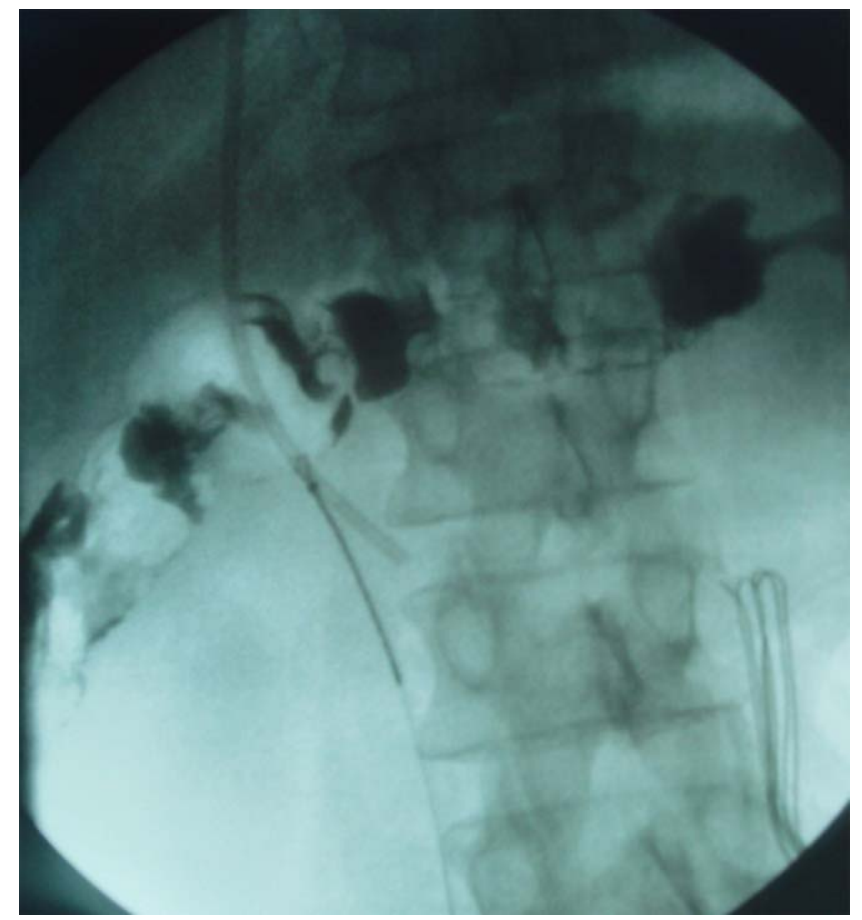

Figura 1 Radiografía intraoperatoria que demuestra la captura del catéter por el sistema de lazo. 


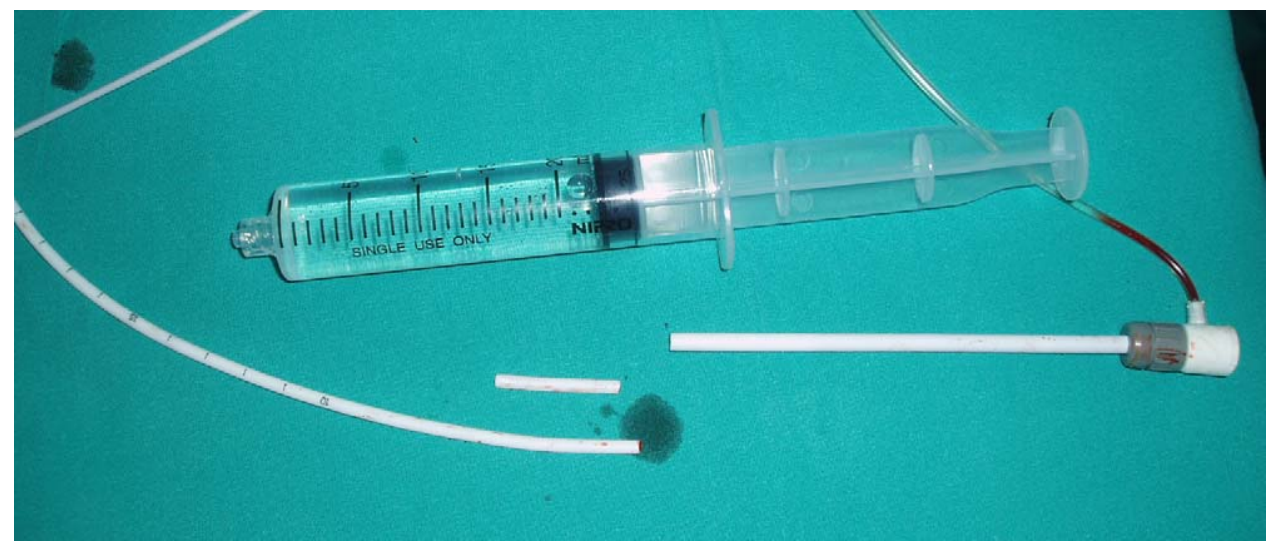

Figura 2 Catéter fracturado y fragmento causado en la retirada percutánea.

introductor el lazo de nitinol dividió de nuevo el fragmento de catéter. A pesar de ello, las dos piezas se pudieron retirar juntas, aunque parte del catéter permaneció fuera de la vaina, pudiendo causar lesión en la pared vascular (fig. 2). El paciente fue dado de alta al día siguiente, sin complicaciones tras un seguimiento a tres meses.

En la práctica médica cada vez es más común la utilización de catéteres venosos centrales. La tasa de ruptura y embolización de un reservorio se estima entre un 0,1 y $1,7 \% 4,5$. Los factores de riesgo son el tipo de catéter utilizado, el lugar de colocación, el tiempo y la sustancia administrada. El primer signo en indicar la dislocación del catéter es el malfuncionamiento de este, pero a veces es un hallazgo casual. Se han descrito complicaciones relacionadas con la embolia de fragmentos, del trombo local, isquemia distal, dolor torácico, arritmias, perforación cardiaca o venosa o incluso riesgo de sepsis grave ${ }^{5}$.

Existen casos en los que los dispositivos intravasculares son stents malposicionados o fragmentados. El riesgo de dañar la pared vascular es elevado. La técnica a utilizar depende de las características del propio stent: flexibilidad, rigidez y fuerza radial $^{6}$.

Se han publicado casos de fragmentos intravasculares con 25 años de permanencia. A los casos de tanto tiempo de evolución se les supone un elevado riesgo de infección y una retirada más difícil por la reacción trombótica e intimal que provocan $^{7}$.

Existen varios dispositivos para la recuperación percutánea de objetos intravasculares, denominados lazos. No se han descrito complicaciones intraoperatorias relacionadas con el procedimiento. Este dispositivo se considera de elección para la extracción de todo tipo de cuerpo extraño intravascular. Varios estudios han evaluado la eficacia y seguridad del sistema de lazo en el tratamiento de esta complicación ${ }^{8,9}$. Koseoglu et al publicaron una serie de 15 pacientes con cuerpos extraños intravasculares como fragmento de reservorios, guías, introductores y embolización de un coil ${ }^{10}$. En ningún caso hubo complicaciones ni se requirió cirugía.
Creemos que el factor más importante para evitar la complicación descrita es utilizar un introductor de diámetro al menos $4 \mathrm{Fr}$ mayor al fragmento a retirar, para evitar nuevas fragmentaciones del mismo en el momento de la extracción percutánea.

\section{Bibliografía}

1. Ramondo $A B$, Favero $L$, Chioin R. Percutaneous retrieval of a broken catheter from the left atrium in an adult. J Interv Cardiol. 2002;15:417-9.

2. Di Carlo I, Fisichella P, Russello D, Puleo S, Latteri F. Catheter fracture and cardiac migration: a rare complication of totally implantable venous devices. J Surg Oncol. 2000;73: 172-3.

3. Yang FS, Ohta I, Chiang HJ, Lin JC, Shih SL, Ma YC. Non-surgical retrieval of intravascular foreign body: experience of 12 cases. Eur J Radiol. 1994;18:1-5.

4. Filippou DK, Tsikkinis C, Filippou GK, Nissiotis A, Rizos S. Rupture of totally implantable central venous access devices (Intraports) in patients with cancer: report of four cases. World J Surg Oncol. 2004;2:36.

5. Kurul S, Saip P, Aydin T. Totally implantable venous-access ports: locally problems and extravasation injury. Lancet Oncol. 2002;3:684-92.

6. Gabelman A, Kramer S, Gorich J. Percutaneous retrieval of lost or misplaced intravascular objects. AJR. 2001;176: 1509-13.

7. Van Der Akker-Berman LM, Pinzur S, Aydinalp A, Brezins M, Gellerman M, Elani A, et al. Uneventful 25 year course of an intracardiac intravenous catheter fragment in the right heart. J Interv Cardiol. 2002;15:421-3.

8. Surov $A$, Jordan $K$, Buerke $M$, Persing $M$, Wollschlaeger $B$, Behrmann C. Atypical pulmonary embolism of port catheter fragments in oncology patients. Support Care Cancer. 2006;25:1-5.

9. Egglin TK, Dickey KW, Rosenblatt M, Pllak JS. Retrieval of intravascular foreign bodies: experience in 32 cases. AJR Am J Roentgenol. 1995;164:1259-64.

10. Koseoglu K, Parildar M, Oran I, Memis A. Retrieval of intravascular foreign bodies with goose neck snare. Eur J Radiol. 2004;49:281-5. 